\title{
24 hour and fractionated profiles of adrenocortical activity in asthmatic patients receiving inhaled and intranasal corticosteroids
}

\author{
Andrew M Wilson, Brian J Lipworth
}

\begin{abstract}
Background-As both rhinitis and asthma are allergic conditions, they frequently occur together. The objective of this study was to assess the diurnal adrenocortical activity in asthmatics receiving inhaled (inh) and intranasal (n) formulations of two different corticosteroids, fluticasone propionate (FP) and triamcinolone acetonide (TAA), both given at clinically recommended doses.

Methods-Twelve stable moderately severe asthmatic subjects of mean age 23.9 years and mean forced expiratory volume in one second $\left(\mathrm{FEV}_{1}\right) \mathbf{8 4 \%}$ predicted were recruited into a randomised placebo (PL) controlled two-way crossover study comparing $\mathrm{nPL}+$ inhPL, $\mathrm{nPL}+\mathrm{inhFP}(880 \mu \mathrm{g}$ bid), and nFP (200 $\mu$ g once daily) + inhFP $(880 \mu \mathrm{g}$ bid) with $\mathrm{nPL}+$ inhPL, $\mathrm{nPL}+$ inhTAA $(800 \mu \mathrm{g}$ bid) and nTAA $(220 \mu \mathrm{g}$ once daily) + inhTAA (800 $\mu$ g bid), each given for five days with a $\mathbf{1 0}$ day washout period. Twenty four hour integrated and fractionated (overnight, 08.00 hours, daytime) serum cortisol levels and urinary cortisol/creatinine excretion were
\end{abstract} measured.

Results-For 24 hour and fractionated serum cortisol levels and corrected urinary cortisol/creatinine excretion there were significant $(p<0.05)$ differences between all active treatments and placebo. For 24 hour integrated serum cortisol levels the ratio between inhaled TAA and FP was 2.3 fold (95\% CI 1.2 to 4.3 ), and for 24 hour urinary cortisol/creatinine excretion the ratio was two-fold (95\% CI 1.2 to 3.4$)$. For 24 hour urinary cortisol excretion, with all active treatments, individual abnormal low values of $<40 \mathrm{nmol}(<14.4 \mu \mathrm{g})$ occurred in 17/24 with FP compared with $4 / 24$ with TAA $(p<0.0005)$. The 24 hour serum cortisol profile was flattened by FP but not with TAA. The addition of nasal corticosteroid did not produce further significant suppression of mean cortisol values, although with intranasal FP there were three more abnormal values for 24 hour urinary cortisol excretion than with inhaled FP alone.

Conclusions-Both inhaled FP and TAA caused significant suppression of adrenocortical activity which was twice as great with FP, the latter being associated with significantly more individual abnormal values and loss of the normal diurnal cir- cadian rhythm. Fractionated serum cortisol levels and urinary cortisol/creatinine excretion were as sensitive as the respective integrated 24 hour measurements. Although the addition of intranasal formulations did not produce further significant suppression of mean values, there were more individual abnormal cortisol values associated with the addition of intranasal FP.

(Thorax 1999;54:20-26)

Keywords: adrenal suppression; asthma; corticosteroids

Asthma and rhinitis are two common conditions which frequently co-exist and are both occurring with increasing prevalence. Topically delivered inhaled (inh) and intranasal (n) corticosteroids are widely recognised to be the first line anti-inflammatory treatment for both conditions. ${ }^{12}$ Although the degree of systemic bioactivity is markedly less than with oral corticosteroids, it is becoming increasingly recognised that inhaled corticosteroids are associated with dose related systemic adverse effects at clinically recommended doses. ${ }^{3}$ Given that these two conditions commonly occur together, it is important to evaluate whether there is any increase in the total systemic burden brought about by the addition of intranasal corticosteroids to patients already taking inhaled corticosteroids.

It is worth considering the different markers for assessing the function of the hypothalamicpituitary-adrenal axis. Adrenal suppression is recognised as a sensitive and reproducible marker of the systemic bioactivity of corticosteroids. ${ }^{4}$ A 24 hour collection for urinary cortisol excretion is a commonly used method for integrating cortisol production and is regarded to be as sensitive as a high dose $(250 \mu \mathrm{g})$ ACTH stimulation test for detecting adrenal suppression in patients taking long term inhaled corticosteroids. ${ }^{5}$ Compliance may be an important factor in collecting a complete 24 hour urine collection, so the use of fractionated urinary cortisol excretion has been suggested to overcome this problem. Overnight or early morning urinary cortisol excretion has been shown to be as sensitive as a 24 hour collection, particularly when corrected for creatinine, in detecting adrenal suppression in healthy subjects receiving inhaled corticosteroids. ${ }^{6}$ Studies in asthmatic patients have also shown overnight urinary cortisol/ creatinine excretion to be extremely sensitive at 
detecting systemic effects of inhaled corticosteroids in adults and children. ${ }^{7-11}$

The aim of this study was therefore to evaluate the integrated 24 hour and fractionated profiles for serum and urinary cortisol in asthmatic patients receiving inhaled corticosteroids given alone or in conjunction with aqueous formulations of intranasal corticosteroids. Triamcinolone acetonide (TAA) and fluticasone propionate (FP) were chosen for investigation as examples of corticosteroids with different pharmacological and pharmacokinetic properties, and both were given within the manufacturer's licensed dose range for inhaled and intranasal formulations.

\section{Methods}

PATIENTS

A random sample of 12 stable moderately severe asthmatic patients were recruited from the adult asthmatic population of Dundee. Entry criteria to the study were that all patients had asthma as defined by the American Thoracic Society, ${ }^{12}$ were receiving inhaled steroids at a dose of up to $1200 \mu \mathrm{g}$ per day (after step down to the lowest maintenance dose), and had a forced expiratory volume in one second $\left(\mathrm{FEV}_{1}\right)$ greater than $70 \%$ predicted. All subjects also had a medical examination, a normal full blood count, biochemical profile (including creatinine, urea and electrolytes, liver function, and bone group tests), and normal urinalysis. Patients were excluded if they had received oral prednisolone within six months of the study. Approval for the study was obtained from the Tayside medical ethics committee and all patients gave written informed consent.

The study sample comprised six men and six women of mean (SE) age 25.9 (3.5) years, mean $\mathrm{FEV}_{1} 84.0$ (4.0)\% predicted, mean mid forced expiratory flow rate $\left(\mathrm{FEF}_{25-75}\right) 56.1$ $(6.0) \%$ predicted, and a median dose of inhaled corticosteroids of $500 \mu \mathrm{g} /$ day (range 200-1200 $\mu \mathrm{g} /$ day). One patient was also receiving intranasal corticosteroid at a dose of $200 \mu \mathrm{g} /$ day.

\section{STUDY DESIGN}

A randomised single (investigator) blind, placebo (PL) controlled, crossover design was used. Patients were randomised in blocks to receive one of two treatment sequences: (1) nPL+inhPL, nPL+inhTAA, nTAA+inhTAA, washout, nPL+inhPL, nPL+inhFP, and $\mathrm{nFP}+$ inhFP or (2) nPL+inhPL, nPL+inhFP, nFP+inhFP, washout, nPL+inhPL, nPL+inhTAA, nTAA+inhTAA. Each patient took both an active or placebo inhaler (eight puffs bid) and an active or placebo nasal spray (two squirts via each nostril once daily) on all 15 days of each arm of the trial.

There was also a 10 day washout period between each of the 15 day treatment sequences during which patients received their usual maintenance inhaled corticosteroid therapy. Prior to each 10 day drug period with the active drug (FP or TAA), patients received the respective matching placebo inhaler and placebo nasal spray for five days (making a total of 15 days for each treatment sequence). The patients' usual inhaled and intranasal corticosteroid therapy was discontinued during the placebo and treatment periods.

The drug treatment phase consisted of five days of active inhaler plus placebo nasal spray, followed by five days of active inhaler plus active nasal spray. Patients were randomised to receive either TAA or FP first. Triamcinolone acetonide was given as Azmacort integrated spacer actuator $100 \mu \mathrm{g}$ per actuation and Nasacort AQ $55 \mu \mathrm{g}$ per actuation (RhonePoulenc Rorer Inc, USA), and fluticasone propionate was given as Flovent metered dose inhaler $110 \mu \mathrm{g}$ per actuation without spacer and Flonase $50 \mu \mathrm{g}$ per actuation (GlaxoWellcome Inc, USA). As each inhaler was given at a dose of eight puffs bid and each nasal spray was two puffs up each nostril once daily, the doses of each drug were as follows: Azmacort $1600 \mu \mathrm{g} /$ day, Nasacort AQ $220 \mu \mathrm{g} /$ day, Flovent $1760 \mu \mathrm{g} / \mathrm{day}$, and Flonase $200 \mu \mathrm{g} /$ day. Both inhaled and intranasal drugs were prescribed according to the manufacturers' recommendations including priming of the nasal sprays. Azmacort oral inhaler has an integrated actuator spacer device, whilst the Flovent metered dose inhaler is not licensed for use with a spacer. The above inhaled corticosteroid doses refer to the dose delivered ex-actuator, in accordance with US product labelling, and are therefore less than the nominal dose (ex-valve). For example, Flovent $110 \mu \mathrm{g}$ per puff exactuator dose is equivalent to $125 \mu \mathrm{g}$ per puff ex-valve dose, whilst Azmacort $100 \mu \mathrm{g}$ per puff ex-actuator/spacer is equivalent to $200 \mu \mathrm{g}$ per puff ex-valve.

The inhalers and nasal sprays were masked and sealed in envelopes by a pharmacist along with instruction sheets at the beginning of the trial in order to make it investigator blind. Although the inhalers for each drug had a different appearance, there were no visible clues as to the identity of the constituent drug. None of the subjects had previously used Flovent, Azmacort, Flonase, or Nasacort formulations. Prior to the study and at each visit subjects were given detailed instructions by a third party on how to use their inhalers and nasal sprays according to the manufacturers' package insert instructions. Co-ordination between inspiration and actuation was also checked at every attendance with the use of a Vitalograph aerosol inhalation monitor device (Vitalograph, Bucks, UK). In addition, instructions were given to discharge inhalers and nasal sprays on each occasion and all inhalations were followed by mouth rinsing. Each subject received a detailed written instruction sheet to follow while taking their inhaler and nasal spray at home and a simple tick chart was used as an aid to compliance.

\section{MEASUREMENTS}

The subjects were admitted to the investigation ward from 19.00 hours on the fourth day of each treatment or placebo phase until 20.30 hours on the fifth day of each treatment or placebo phase. Subjects continued to take their study medication whilst in the investigation 
ward. On arrival at the ward a cannula was inserted into the antecubital fossa vein to permit blood sampling and was flushed with heparinised saline with dead space removal prior to each sampling. Blood samples were taken for measurement of serum cortisol levels at 20.00, 24.00, 04.00, 08.00, 12.00, 16.00, and 20.00 hours. The subjects rested, lying supine, for at least 30 minutes prior to each blood sample. All blood samples were taken within a five minute window for each time point.

Subjects emptied their bladder at 19.30 hours on the fourth day of the treatment or placebo (immediately prior to lying supine before the first serum sample), and all urine was collected from 20.00 hours until 07.30 hours (immediately prior to lying supine before the fourth serum sample was taken). The patients were encouraged to drink freely on waking and were requested to pass a further sample of urine at 08.00 hours. This subsequently became incorporated as the start of the daytime sample. Patients were asked to finally empty their bladder at 20.00 hours to finish the daytime collection (08.00-20.00 hours). The total volumes of the overnight, 08.00 hours, and daytime urine collections were measured. Aliquots were kept for assay of cortisol and creatinine levels. The 24 hour value was derived from the sum of the two 12 hour samples (daytime and overnight).

Spirometric values were also measured using a Vitalograph Compact spirometer (Vitalograph Ltd, Buckingham, UK) at each visit, although efficacy was not intended as an end point due to the short duration of treatment.

We did not perform an ACTH (Cosyntropin) stimulation test because Synacthen (Novartis, UK) is contraindicated on the UK data sheet for use in atopic and asthmatic patients due to reports of anaphylactic reactions, particularly with repeated administration. It was therefore considered unethical on safety grounds to perform an ACTH stimulation test for research purposes only.

ASSAYS

All assays were performed in duplicate and in a blinded fashion by a separate technician. Serum cortisol levels and urinary cortisol excretion were measured using a commercial radioimmunoassay (RIA) kit with no cross reactivity for either fluticasone propionate or triamcinolone acetonide (Immunodiagnostic Systems Ltd, Boldon, Tyne \& Wear, UK). The coefficient of variation (CV) for analytical imprecision for urinary cortisol excretion within the assay was $10 \%$ and between the assays was $7.2 \%$. Corresponding $\mathrm{CV}$ values for serum cortisol measurements were $7.1 \%$ for within assay and $7.2 \%$ for between assay. Urinary creatinine excretion was measured on a Cobas-Bio autoanalyser (Roche Products Ltd, Welwyn Garden City, UK) with an intraassay $\mathrm{CV}$ of $3.9 \%$ and an inter-assay $\mathrm{CV}$ of $0.63 \%$.
STATISTICAL ANALYSIS

The study was designed with a sample size of 12 with $80 \%$ power (beta error $=0.2$ ) to detect a $20 \%$ difference in serum cortisol levels (the primary end point) between treatments with the alpha error set at 0.05 (two tailed). In order to normalise the distribution all data were log transformed prior to analysis and all values are therefore quoted as geometric means. The area under the curve (AUC) was generated from the integrated 24 hour serum sample profile using the trapezoidal method and the fractionated (overnight, 08.00 hours, daytime) moieties were also analysed separately. The fractionated (overnight, 08.00 hours, daytime) and 24 hour urinary cortisol collections were analysed after correcting for urinary creatinine excretion. All data were analysed using a Statgraphics software package (STSC Software Group, Rockville, Maryland, USA).

Comparisons between all four active treatments and both placebos were made by a multifactorial overall analysis of variance with subject, drug, treatment, and period as factors. Bonferroni's multiple range testing was then applied to assess where there were significant differences between treatments. The Bonferroni's multiple range test was set with $95 \%$ confidence intervals and hence any significant differences between treatments are reported only at the $p<0.05$ level. For all end points $95 \%$ confidence intervals were calculated for the ratios of geometric means between TAA and FP. A comparison was also made between the first and second placebos given in sequence within the study design to check for any carryover effect between the two drug sequences. In addition, a comparison was made of the two placebos given before FP and TAA, irrespective of the treatment sequence.

Individual values for low 24 hour urinary cortisol excretion of $<40 \mathrm{nmol}(<14.4 \mu \mathrm{g})$ and 08.00 hours serum cortisol levels of $<150 \mathrm{nmol} / 1(<5.4 \mu \mathrm{g} / \mathrm{dl})$ were analysed using the $\chi^{2}$ test. Values of $<40 \mathrm{nmol}$ for 24 hour urinary cortisol excretion and $<150 \mathrm{nmol} / 1$ for 08.00 hours serum cortisol level are considered to be abnormal values below the reference range. ${ }^{13}$

\section{Results}

There were no significant carryover effects between the first and second placebos given in sequence using any of the parameters measured (figs 1, 2, 5). Placebo values prior to treatment (irrespective of sequence) with either FP or TAA were also not significantly different (FP vs TAA): (a) 24 hour serum cortisol 6880 vs $7280 \mathrm{nmol} . \mathrm{h} / \mathrm{l}$; (b) fractionated serum cortisol (overnight, 2590 vs $2760 \mathrm{nmol} . \mathrm{h} / \mathrm{l} ; 08.00$ hours, 587 vs $608 \mathrm{nmol} . \mathrm{h} / \mathrm{l}$; daytime, 4240 vs $4460 \mathrm{nmol} . \mathrm{h} / \mathrm{l}$ ); (c) 24 hour corrected urinary cortisol/creatinine excretion 7.0 vs $6.4 \mathrm{nmol} /$ mmol; or (d) fractionated urine collections corrected for creatinine (overnight, 3.9 vs 3.8 $\mathrm{nmol} / \mathrm{mmol}$; 08.00 hours, 16.1 vs $16.5 \mathrm{nmol} /$ mmol; daytime, $9.3 \mathrm{vs} 9.1 \mathrm{nmol} / \mathrm{mmol}$ ). Mean $\mathrm{FEV}_{1}$ values showed a significant difference between both placebo and inhaled alone for both treatments, but no difference between the 


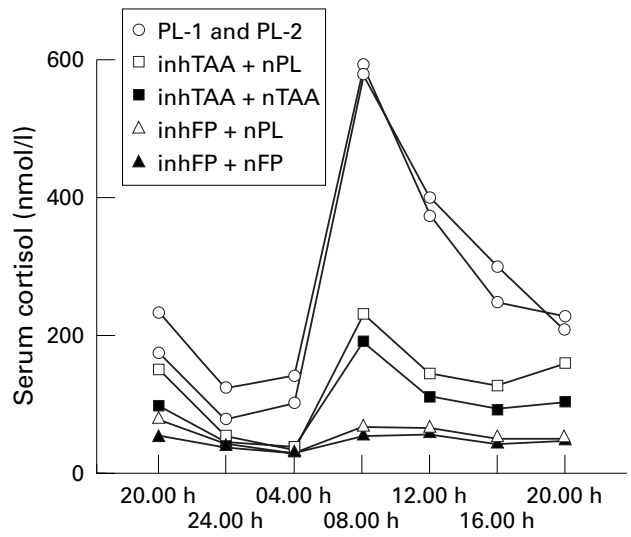

Figure 1 Geometric means for 24 hour profile of serum cortisol levels for first and second placebo (PL-1 and PL-2), inhaled triamcinolone acetonide with nasal placebo (inhTA $A+n P L)$, inhaled fluticasone propionate with nasal placebo (inhFP+nPL), inhaled plus nasal triamcinolone acetonide (inhTA $A+n T A A)$, and inhaled plus nasal fluticasone propionate (inhFP $+n F P)$.

two drugs for either inhaled alone or combined inhaled and intranasal administration: PL: 2.981 , inhTAA + nPL: 3.181 , inhFP + nPL: 3.29 l, inhTAA + nTAA: 3.05 l, inhFP + nFP: 2.941 .

SERUM CORTISOL

Inspection of the 24 hour serum cortisol time profile shows that the normal diurnal circadian rhythm was abolished by FP (fig 1). With TAA there was blunting of the 08.00 hours early morning cortisol peak although the normal diurnal circadian rhythm remained preserved. For both 24 hour and fractionated serum cortisol levels there was a significant difference $(p<0.05)$ between placebo and all of the other
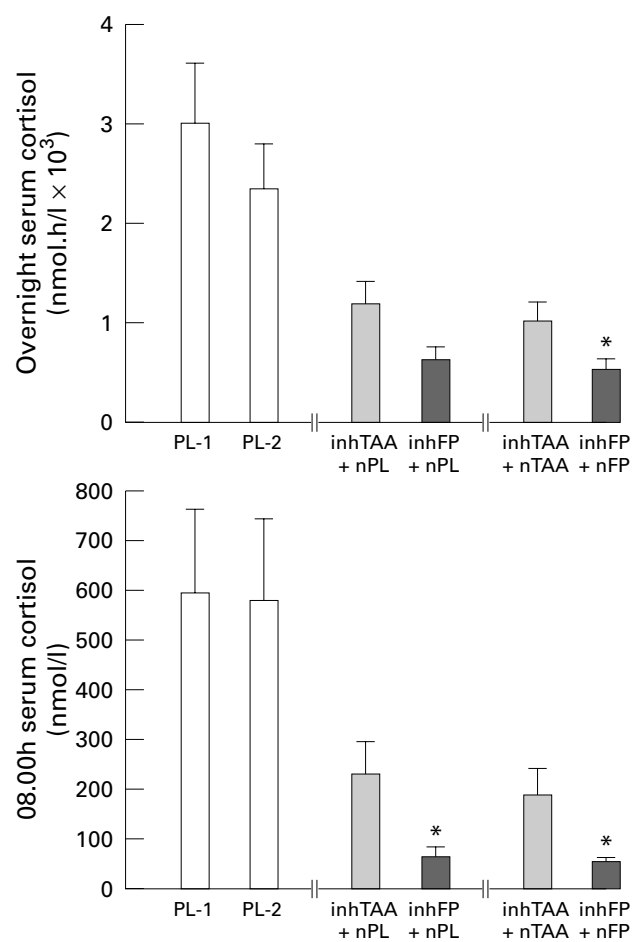

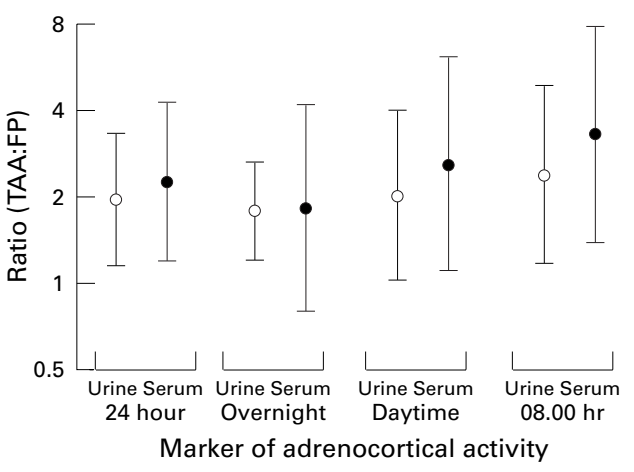

Figure 3 Inhaled TAA:FP ratio (with 95\% confidence intervals) for 24 hour, overnight, daytime and 08.00 hours urinary cortisol/creatinine excretion ( 0 ) and serum cortisol levels (•). 95\% confidence intervals which exclude unity indicate a significant difference between the two drugs (plotted on a $\log _{2}$ scale).

four active treatments. There was a significant difference $(p<0.05)$ between FP and TAA for both inhaled medication alone and inhaled plus nasal medication (figs 2 and 3). For serum cortisol levels at 08.00 hours with all active treatments there was a significant difference $(\mathrm{p}<0.0005)$ in the number of individual values between FP (17/24 (71\%)) and TAA (3/24 $(12 \%)$ ) with an abnormally low level of $<150 \mathrm{nmol} / 1$ (fig 4). The addition of intranasal corticosteroid did not produce any further significant suppression of mean serum cortisol values.

\section{URINARY CORTISOL/CREATININE}

For 24 hour and fractionated measurements there was a significant difference $(p<0.05)$ between placebo and all of the other active treatments and a significant difference
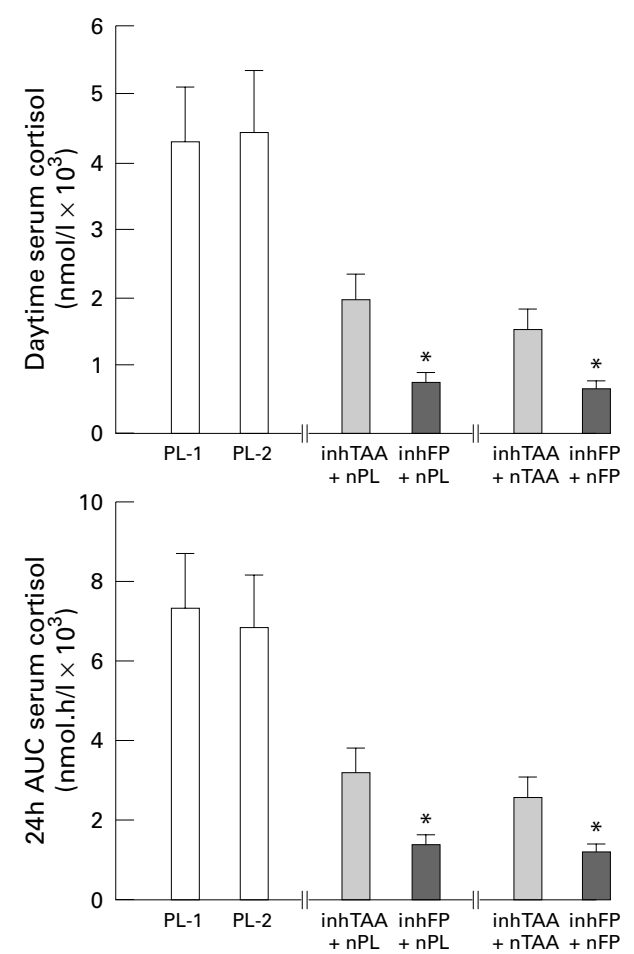

Figure 2 Geometric means with standard error for 24 hour and fractionated serum cortisol levels. There was a significant $(p<0.05)$ difference between all four active treatments and both placebos. Asterisks denote a significant $(p<0.05)$ difference between FP and TAA for inhaled alone or inhaled plus nasal medication. Abbreviations as in fig 1 . 

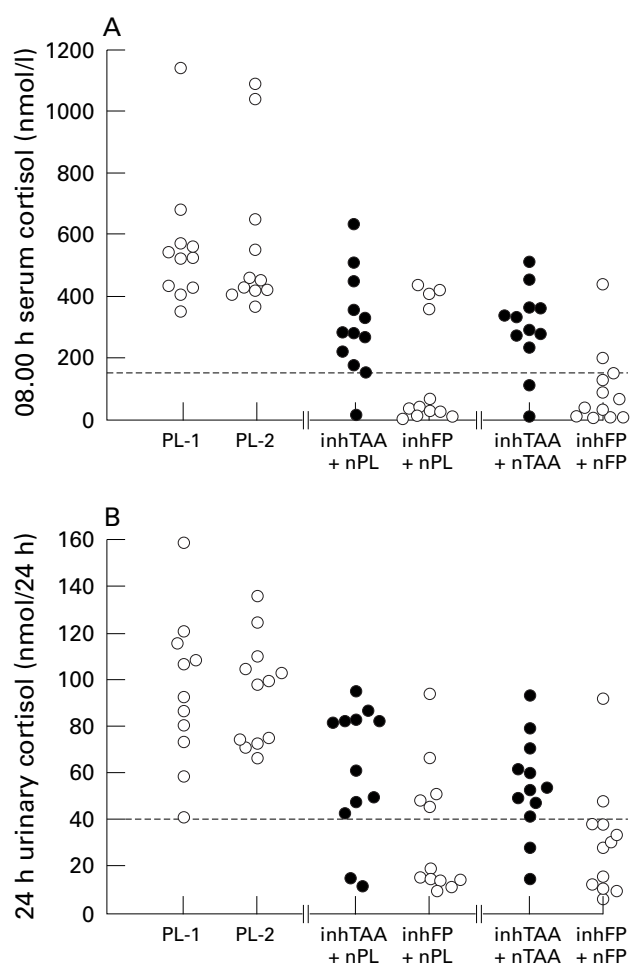

Figure 4 Individual values for $(A)$ serum cortisol levels at 08.00 hours and (B) 24 hour urinary cortisol excretion. For all four active treatments the numbers of individual patients with an abnormal serum cortisol level of $<150 \mathrm{nmol} / \mathrm{l}(<5.4 \mu \mathrm{g} / \mathrm{dl})$ at 08.00 hours were: FP 17/24 (71\%), and TAA $3 / 24(12 \%)(p<0.0005)$. The corresponding numbers of individual patients with an abnormal 24 hour urinary cortisol excretion of $<40 \mathrm{nmol}$ $(<14.4 \mu \mathrm{g})$ were: $F P$ 17/24 (71\%) and TAA 4/24 (16\%) $(p<0.0005)$. Abbreviations as in fig 1 .
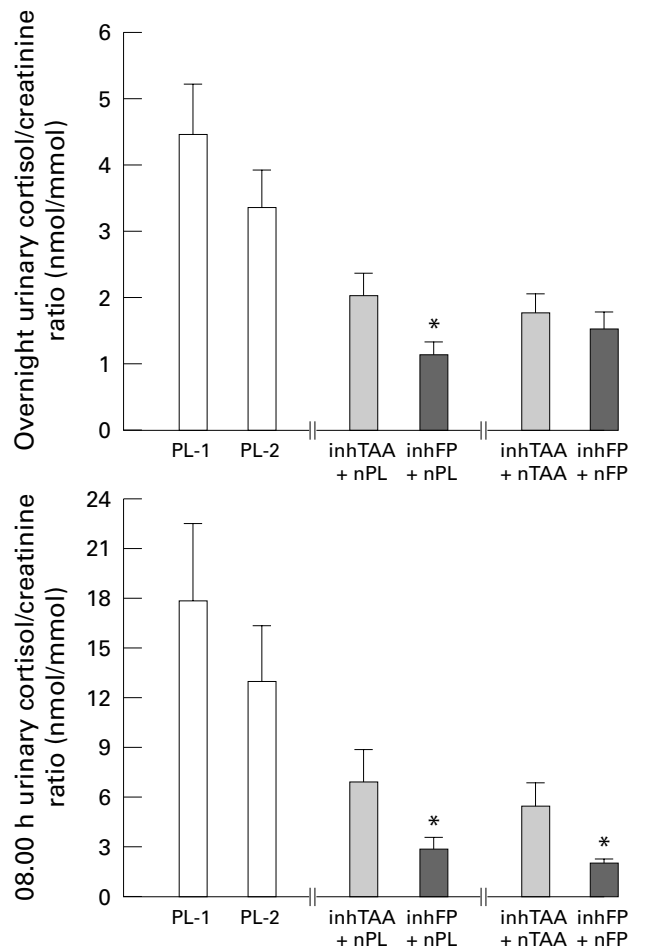

$(\mathrm{p}<0.05)$ between FP and TAA for both inhaled medication alone and inhaled plus nasal medication (figs 3 and 5). The 95\% CI for within subject differences showed less variance for urine than for serum measurements (fig 3). For 24 hour urinary cortisol excretion (fig 4) there was a significant difference $(\mathrm{p}<0.0005)$ between those treated with FP $(17 / 24(71 \%))$ and TAA $(4 / 24(16 \%))$ when analysing the number of individual values with an abnormally low level of $<40 \mathrm{nmol}$. The addition of nasal corticosteroid did not produce any further significant suppression of mean urinary cortisol/creatinine values. However, the addition of intranasal FP resulted in three more abnormal values for 24 hour urinary cortisol excretion compared with inhaled FP alone (fig 4).

\section{Discussion}

We have shown for 24 hour and fractionated measurements of serum cortisol levels and corrected urinary cortisol/creatinine excretion that both inhaled FP and TAA produced significant adrenal suppression compared with placebo. Inhaled FP also produced significantly greater suppression than inhaled TAA, amounting to a two fold difference between the drugs. As expected, the 24 hour cortisol profile for placebo showed that the serum cortisol peak occurs at 08.00 hours which coincides with the largest difference between treatments. This reinforces the importance for precise timing of the early morning serum cortisol samples at 08.00 hours rather than the use of a variable 08.00-10.00 hours window as in previous multicentre studies. ${ }^{14-16}$ Although both inhaled drugs produced a significant improvement in
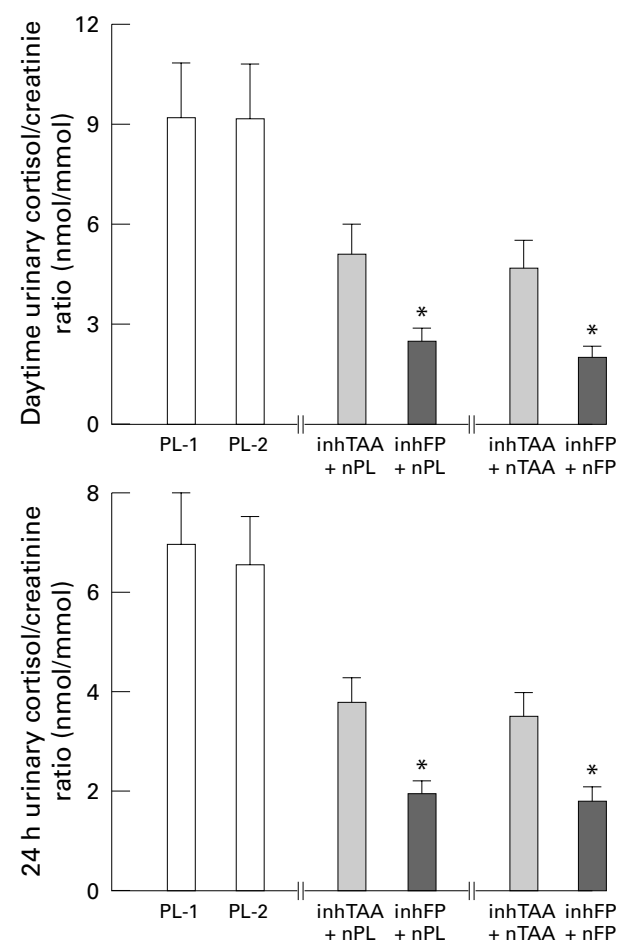

Figure 5 Geometric means with standard error for 24 hour corrected urinary cortisol/creatinine excretion and fractionated (overnight, 08.00 hours, and daytime) corrected urinary cortisol/creatinine. Asterisks denote a significant $(p<0.05)$ difference between TAA and FP for inhaled or inhaled plus nasal medication. There was a significant difference between all four active treatments and both placebos for 24 hour and fractionated measurements. Abbreviations as in fig 1. 
$\mathrm{FEV}_{1}$ after five days, conclusions cannot be drawn regarding relative anti-asthmatic efficacy as this would require a much longer duration of treatment. We did not attempt to compare therapeutically equivalent doses of both drugs as there are no proper dose-response data for the relative efficacy of FP and TAA for effects in asthma and allergic rhinitis. Thus, from our data it is not possible to conclude the relative therapeutic index for FP and TAA when given by their respective inhaled and intranasal formulations. The doses were chosen to represent the highest licensed for TAA (as Azmacort) and FP (as Flovent) in the USA for both inhaled and intranasal formulations. A dose delivered ex-actuator of Flovent $110 \mu \mathrm{g}$ per puff is equivalent to $125 \mu \mathrm{g}$ per puff as the nominal dose ex-valve. The Flovent product information recommends that the highest dose of $1760 \mathrm{mg}$ per day be used for severe asthmatic patients requiring maintenance oral corticosteroids.

As there is no first pass metabolism of FP or TAA via the nose or lung but extensive hepatic first pass metabolism, the total systemic bioavailability is predominantly determined by the respective nasal and lung absorption. The greater degree of intranasal deposition compared with lung deposition will be compensated by the smaller nasal surface area along with nasociliary clearance into the throat, all of which will determine the relative systemic bioavailability from lung and nose. Indeed, we have recently shown that, when given alone, aqueous formulations of intranasal FP $200 \mu \mathrm{g} /$ day and TAA $220 \mu \mathrm{g} /$ day produced mean values of $43 \%$ and $23 \%$ adrenal suppression, respectively. ${ }^{17}$

The results expressed as mean values provide quantitative information on the statistical significance for an average patient, but may be misleading as there is a wide interindividual variability in the systemic response as evidenced by the dispersion of individual values. This may represent individual differences in bioavailability or possibly effects of glucocorticoid receptor responsiveness. ${ }^{18}$ It is therefore more relevant for the clinician to consider the response in terms of individual levels which are below the normal reference range. It is evident from inspection of the scatterplots for 08.00 hours serum cortisol levels and 24 hour urinary cortisol excretion that the majority of patients receiving inhaled FP alone or with intranasal FP had suppression below that accepted as normal. ${ }^{13}$ In contrast, there were only a few abnormal individual low values for inhaled TAA alone or with intranasal TAA. Although the mean data showed no significant additive suppression with intranasal $\mathrm{FP}$, it was evident from the individual data for 24 hour urinary cortisol excretion that there were a further three abnormal values with combination therapy compared with inhaled FP alone. This, in turn, suggests that the bioavailability from the nasal moiety may contribute to the overall systemic burden in certain susceptible patients. ${ }^{17}$ In this respect it is known that patients receiving inhaled corticosteroids who have an attenuated cortisol response to ACTH stimulation also have a suppressed 24 hour urinary cortisol excretion. ${ }^{5}{ }^{18-20}$ It was considered unethical by the medical ethics committee to administer ACTH (Cosyntropin) to our patients for research purposes as it is contraindicated on the UK data sheet (Synacthen, Novartis, UK) for use in atopic or asthmatic subjects due to reports of anaphylactic reactions, particularly with repeated administration.

The observed differences between TAA and FP can be explained by the relative pharmacological and pharmacokinetic properties of both drugs. $^{21}$ Fluticasone propionate has greater glucocorticoid potency, longer receptor residency time, and is more lipophilic, resulting in greater systemic tissue retention and blood accumulation. It is also important to consider the lung delivery and, in this respect, the respirable fraction of the TAA integrated spacer is $69 \%$ compared with $34 \%$ for the FP metered dose inhaler. ${ }^{22}{ }^{23}$ Thus, it is likely that the systemic bioactivity of FP would be enhanced if it was given via a metered dose inhaler with spacer device.

The results of this study showing significant suppression of overnight and early morning moieties of urinary cortisol excretion are comparable to our previous findings with TAA $1600 \mu \mathrm{g} /$ day given to healthy volunteers. ${ }^{11} \mathrm{We}$ observed that fractionated moieties of serum cortisol and urinary cortisol/creatinine were as sensitive as integrated 24 hour measures, although the intra-individual variability was greater for serum levels than for urinary cortisol excretion (for between treatment differences as $95 \% \mathrm{CI}$ ). Indeed, the largest signal to noise ratio was observed for the difference in overnight urinary cortisol/creatinine excretion. In order to overcome the variability in a spot serum sample of cortisol at 08.00 hours, 24 hour collections of urinary cortisol excretion have been used to assess diurnal adrenocortical activity and have been shown to be more sensitive. ${ }^{18}$ Furthermore, 24 hour urinary cortisol excretion correlated well with 24 hour serum cortisol levels in detecting adrenal suppression with inhaled FP in healthy volunteers. ${ }^{24}$ Fractionated overnight and early morning urine samples have previously been considered to be as sensitive as a 24 hour sample, evidenced by McIntyre et al in a study of healthy volunteers taking $800 \mu \mathrm{g} /$ day and $2000 \mu \mathrm{g} /$ day of inhaled beclomethasone dipropionate for 10 days. ${ }^{6}$ We have also shown overnight urinary cortisol/creatinine excretion to be more sensitive than serum cortisol levels at 08.00 hours in previous studies. ${ }^{7911}$ Our results for corrected urinary cortisol/creatinine in this study agree with those of McIntyre et al as fractionated collections were as sensitive as an integrated 24 hour collection.

In conclusion, we have found that both inhaled FP and TAA caused significant suppression of adrenocortical activity which was twice as great with FP as with TAA. With FP, but not with TAA, there was loss of the normal diurnal circadian rhythm which was associated with abnormal low values in the majority of individuals. Fractionated serum cortisol and 
urinary cortisol/creatinine were as sensitive as integrated 24 hour measures, although the intra-individual variability was less for urine than for serum. Although the addition of intranasal formulations did not produce significant further suppression of mean values with the doses used in this study, there were more individual abnormal cortisol values associated with the addition of intranasal FP.

The authors wish to thank Rhone-Poulenc-Rorer for their financial assistance with this study.

1 British Thoracic Society. The British guidelines on asthma management 1995 review and position statement. Thorax 1997; 52(Suppl 1):S1-28.

2 Mind N Glucocorticosteroids and rhinitis. Allergy 1993;48:476-90.

3 Lipworth BJ. Airway and systemic effects of inhaled corticosteroids in asthma: dose response relationship. Pulmono Pharmacol 1996;9:19-27.

4 Hanania NA, Champman KR, Kesten S. Adverse effects of inhaled corticosteroids. Am F Med 1995;98:196-208.

5 Brown PH, Blundell G, Greening AP, et al. Screening for hypothalmo-pituitary-adrenal axis suppression in asthmatics taking high doses of inhaled corticosteroids. Respir Med 1991;85:511-6.

6 McIntyre HD, Mitchell CA, Bowler SD, et al. Measuring the systemic effects of inhaled beclomethasone: timed morning urine collections compared with 24 hour specimens. Thorax 1995;50:1280-4.

7 Wilson AM, McFarlane LC, Lipworth BJ. Dose response effect for adrenal suppression with repeated twice daily inhaled fluticasone propionate and triamcinolone acetonide in adult asthmatics. Am 7 Respir Crit Care Med 1997; nide in adult

8 Clark DJ, Grove A, Cargill RI, et al. Comparative adrenal suppression with inhaled budesonide and fluticasone propionate in adult asthmatic patients. Thorax 1996;51: $262-6$

9 Clark DJ, Lipworth BJ. Adrenal suppression with chronic dosing of fluticasone propionate compared with budesonide in adult asthmatic patients. Thorax 1997;52:55-8

10 Clark DJ, Clark RA, Lipworth BJ. Adrenal suppression with inhaled budesonide and fluticasone propionate given by large volume spacer to asthmatic children. Thorax 1996;51: $941-3$
11 Wilson AM, McFarlane LC, Lipworth BJ. Effects of low and high doses of flunisolide and triamcinolone acetonide on basal and dynamic meausures of adrenocortical activity in healthy volunteers. F Clin Endocrinol Metab 1998;83:922-5.

12 American Thoracic Society. Standards for the diagnosis and care of patients with chronic obstructive pulmonary disease (COPD) and asthma. Am Rev Respir Dis 1987;136:225-43.

13 Honour JW. Hypothalamic-pituitary-adrenal axis. Respir Med 1994;88(Suppl A):9-15.

14 Barnes NC, Marone G, Di Maria GV, et al. A comparison of fluticasone propionate $1 \mathrm{mg}$ daily, with beclomethasone dipropionate $2 \mathrm{mg}$ daily, in the treatment of severe asthma. Eur Respir F 1993;6:877-84.

15 Fabbri L, Burge PS, Croonenburgh L, et al. Comparison of fluticasone propionate with beclomethasone dipropionate in moderate to se

6 Ayres JG, Bateman ED, Lundback B, et al. High dose fluticasone propionate, $1 \mathrm{mg}$ daily, versus fluticasone propionate $2 \mathrm{mg}$ daily or budesonide $1.6 \mathrm{mg}$ daily, in patients with chronic severe asthma. Eur Respir F 1995;8:579-86.

17 Wilson AM, McFarlane LC, Lipworth BJ. Effects of repeated once daily dosing of three intranasal corticosteroids on basal and dynamic measures of HPA-axis activity. F Allergy Clin Immunol 1998;101:470-4.

18 Lipworth BJ, Seckl JR. Measures for detecting systemic bioactivity with inhaled and intranasal corticosteroids. Thorax 1997;52:476-82.

19 Broide J, Soferman R, Kivity S, et al. Low-dose adrenocorticotropin test reveals impaired adrenal function in patients taking inhaled corticosteroids. F Clin Endocrinol Metab 1995;80:1243-6.

20 Grebe SKG, Feek CM, Durham JA, et al. Inhaled beclomethasone dipropionate suppresses the hypothalamic pituitary-adrenal axis in a dose dependent manner. Clin Endocrinol 1997;47:297-304.

21 Lipworth BJ. Pharmacokinetics of inhaled drugs. Br 7 Clin Pharmacol 1996;42:697-705.

22 Iula AK, Flynn C, Deluccia F. Comparative study of the in vitro dose delivery and particle size distribution characteristics of an Azmacort metered dose inhaler in combination with few different spacer devices. Curr Ther Res 1997;58: 644-54.

23 Olsson BO. Aerosol particle generation from dry powder inhalers: can these equal pressurised metered dose inhalers. f Aerosol Med 1995;8(Suppl 3):S13-18.

24 Grahnen A, Eckernas SA, Brundin RM, et al. An assessment of the systemic activity of single doses of inhaled fluticasone propionate in healthy volunteers. $\mathrm{Br} f \mathrm{Clin}$ Pharmacol 1994;38:521-5 